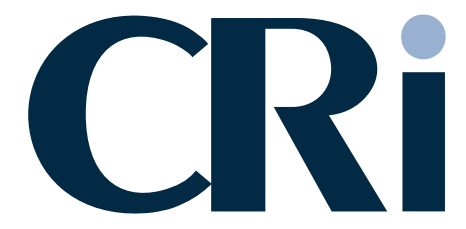

Issue 6

15 December 2005

P. $161-192$
A Journal of Information Law and Technology

\title{
Articles
}

Andrew Manning/John Selby - A Fine Line Between Legal Access And Circumvention

John Beardwood/Daniel Fabiano - Approaches to "ExtraJurisdictional" Data Transfers in Canadian and European Outsourcing

\section{Case Law}

Australia: Circumvention of Technical Protection Measures Stevens v Kabushiki Kaisha Sony Computer Entertainment [2005] HCA 58

USA: Interception of E-Mails in Temporary And Transient EStorage United States of America v. Bradford C. Councilman [No. 03-1383] with remarks by Westermeier

\section{Updates}

Ray Nimmer - USA: Shrink-Wrap License Not Preempted

By Fair Use

Antti Seppälä - Finland: Copyright Act Amended

Ioannis Iglezakis - Greece: Confidentiality of E-Communications

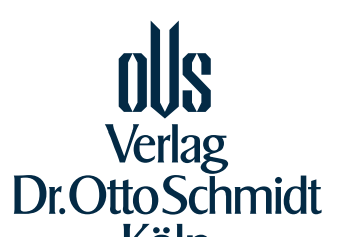

Köln 
New at www cr-international.com

WSIS's initiative for UN Internet Governance Forum of 15 November 2005

\section{Computer Law Review Internationa1}

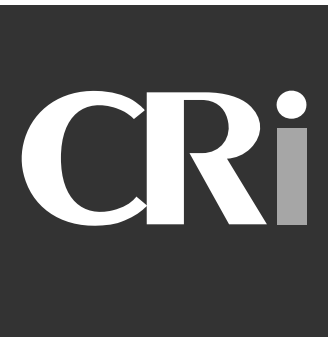

A Journal of Information Law and Technology

6th Year · Issue 6/2005 · Pages 161-192

\section{Editorial Board}

Prof Dr. Thomas Dreier, M.C.J.

University of Karlsruhe

Dr. Jens-L. Gaster, principal administrator, Brussels

RA Thomas Heymann, Frankfurt/M.

Prof. Dr. Michael Lehmann, Dipl-Kfm

Max-Planck-Institute and University of Munich

Prof. Raymond T. Nimmer, University of Houston

Attorney at Law Holly K. Towle, J.D., Seattle

Attorney at Law Thomas Vinje, Brussels

\section{Articles}

Andrew Manning/John Selby - A Fine Line Between Legal Access And Circumvention The Australian High Court provides a pyrrhic victory for the sale of mod-chips

John Beardwood/Daniel Fabiano - Approaches to "Extra-Jurisdictional" Data Transfers in Canadian and European Outsourcing: A Comparative Approach

\section{Case Law}

Australia: Circumvention of Technical Protection Measures Stevens v. Kabushiki Kaisha Sony Computer Entertainment [2005] HCA 58 - High Court of Australia - Decision of 6 October 2005

USA: Interception of E-Mails in Temporary And Transient E-Storage United States of America v. Bradford C. Councilman [No. 03-1383] - Court of Appeals for the $1^{\text {st }}$ Circuit - Decision of 11 August 2005 with remarks by Westermeier

USA: Website Terms of Use Binding for Spider Operators Cairo, Inc. v. Crossmedia Services, Inc. [No. C 04-04825 JW] - District Court for the Northern District of California - Decision of 1 April 2005 with remarks by Westermeier

The Netherlands: Liability for Software Circumventing Technical Protection Measures Stichting BREIN v. DIRECTWAREHOUSE B.V. and Paramount Pictures Cooperation v. DIRECT WAREHOUSE B.V. - President District Court Rotterdam - Decision of 21 July 2005 - Summary \& Comment by Willems

\section{Updates}

Ray Nimmer - USA: Shrink-Wrap License Not Preempted By Fair Use

Antti Seppälä - Finland: Copyright Act Amended 189

loannis Iglezakis - Greece: Confidentiality of E-Communications

- About the Authors

- Masthead

- CRI-Reader Services 


\section{B UCH - T IP P}

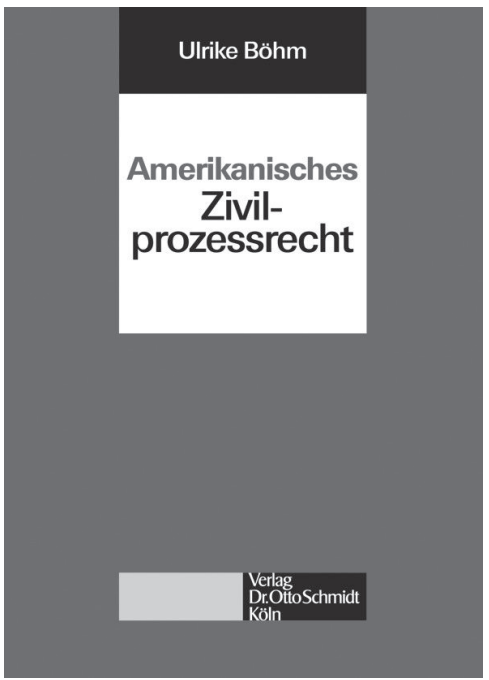

Böhm Amerikanisches Zivilprozessrecht Von Ulrike Böhm. Ca. 420 Seiten DIN A5, 2005, brosch. 39,80€ [D]. ISBN 3-504-65309-4

Je intensiver sich die Wirtschafts- und Rechtsbeziehungen zwischen Deutschland und den USA entwickeln, desto größer wird auch das Prozessrisiko. Vor allem Unternehmensjuristen mit Geschäftsverbindungen in die USA, aber auch Rechtsanwälte, Richter und Referendare sollten daher Bescheid wissen, wie ein amerikanischer Zivilprozess abläuft.

Das Werk gibt eine praxisbezogene und verständliche Anleitung, die bei der Zusammenarbeit mit amerikanischen Kollegen und bei der Mandantenberatung Unterstützung leistet. Es werden Bereiche erläutert, die im Prozess große Bedeutung haben, wie das Discovery-Verfahren oder die Darstellung der Jury Trial. Weitere Kapitel sind u.a. die Forumswahl und Urteilsanerkennung, die wichtige Rolle des Anwalts vor und im Prozess, das Gerichtsverfassungssystem, die Jurisdiction, die Verfahrensbeendigung und die Urteilsanfechtung bzw. -wirkung. Die praktische Bedeutung der Rechtsprechung wird dabei immer wieder besonders hervorgehoben.

Profitieren Sie von den Erfahrungen und dem Wissen der Frankfurter Anwältin Ulrike Böhm (LL.M., New York), die an einem amerikanischen Gericht (Judicial Clerkship) und später in einer amerikanischen Großkanzlei (Attorney at Law) tätig war.

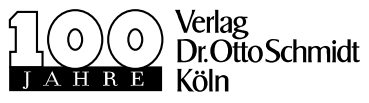

Wir machen Wissen praktikabel

Bestellen Sie bei Ihrer Buchhandlung oder direkt beim Verlag: Fax 0221/9 37 38-943

Gekauft! Ich bestelle mit 14-tägigem Rückgaberecht Böhm Amerikanisches Zivilprozessrecht brosch. 39,80 € [D]. ISBN 3-504-65309-4

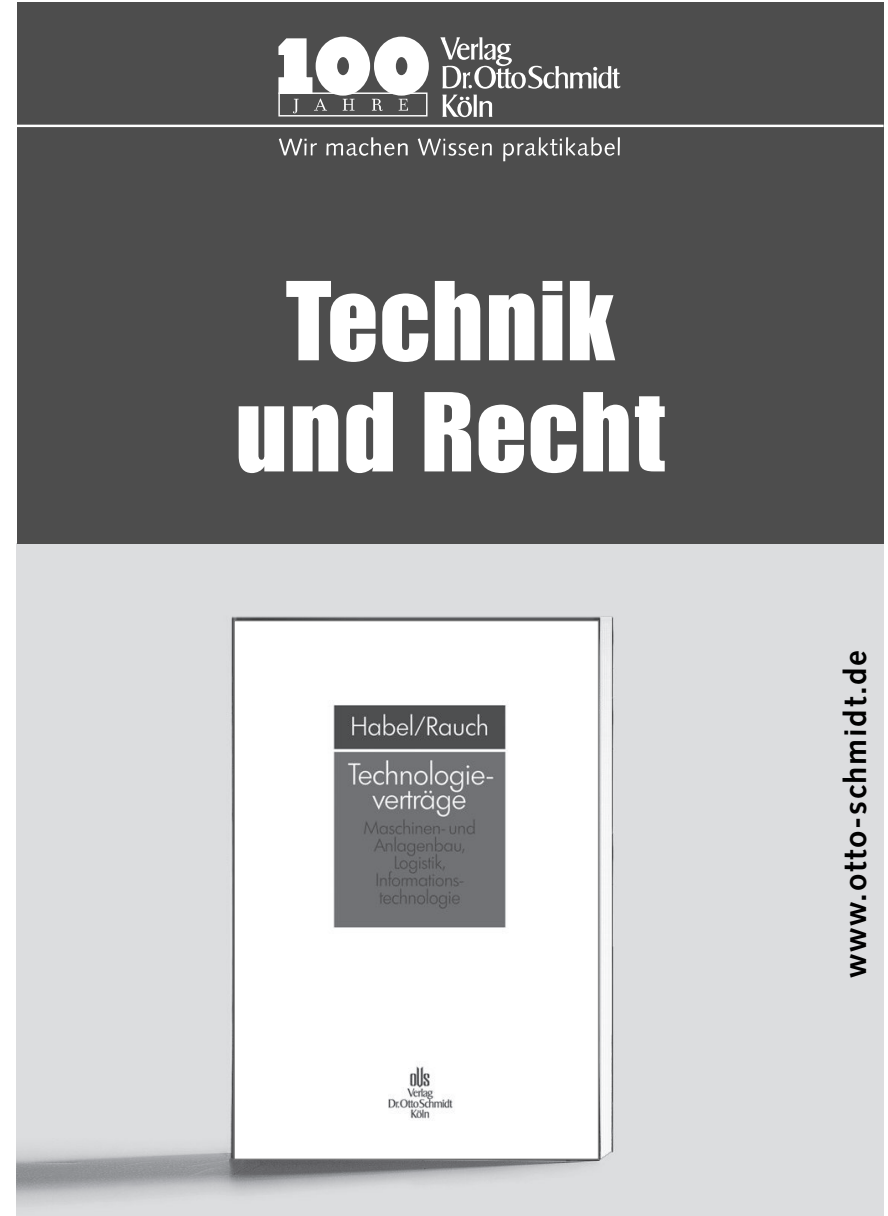

Habel/Rauch, Technologieverträge

Maschinen- und Anlagenbau, Logistik, Informationstechnologie Von RA Dr. Oliver Habel und Max Rauch. 2. Auflage 2005,

376 Seiten, DIN A4, brosch. 139,- € [D]. ISBN 3-504-56046-0

Die Gestaltung von Verträgen mit stark technologieorientiertem Einschlag ist komplex und kompliziert. Die Regelungsbereiche sind so umfangreich, dass schnell der Überblick zwischen Auftragsbeschreibung, Überwachung, Abnahme und Inbetriebnahme verloren geht.

Hier hilft der "Habel/Rauch" mit einem umfangreichen, knapp 200-seitigen Vertragsmuster, das nahezu alle Probleme erfasst und, in einer zweiten Textspalte, kommentiert. So werden alle Formulierungen nachvollziehbar und eröffnen Möglichkeiten der eigenen Gestaltung.

Ein Serviceteil mit über 30 konkreten Arbeitshilfen macht das Werk für alle, die technologieorientierte Projekte begleiten, unverzichtbar.

Bestellen Sie bei Ihrer Buchhandlung oder beim Verlag Dr. Otto Schmidt, Köln

BESTELLSGHEIN Fax: 0221 / 937 38-943

X Ich bestelle mit 14-tägigem Rückgaberecht

Habel/Rauch, Technologieverträge

brosch. 139,- $€[D]$. ISBN 3-504-56046-0

Name

Straße

PLZ / Ort

Datum / Unterschrift 